\title{
Noncardiac Lebanese hospitalized adult patients' awareness of their coronary artery disease risk factors
}

This article was published in the following Dove Press journal: Vascular Health and Risk Management

\author{
Fatima Ghaddar' \\ Pascale Salameh ${ }^{1-3}$ \\ Nadine Saleh ${ }^{1,2}$ \\ Firas Farhat ${ }^{4}$ \\ Ramez Chahine ${ }^{5}$ \\ Nathalie Lahoud ${ }^{1-3,6}$ \\ Mira Hleyhel ${ }^{1,2,6}$ \\ Rouba K Zeidan ${ }^{1,2,6}$
}

'Faculty of Public Health II, Lebanese University, Fanar, Lebanon; ${ }^{2} \mathrm{National}$ Institute of Public Health, Clinical Epidemiology and Toxicology, Faculty of Public Health, Lebanese University, Fanar, Lebanon; ${ }^{3}$ Laboratory of Epidemiological and Clinical Research, Lebanese University, Beirut, Lebanon; ${ }^{4}$ Faculty of Medical Sciences, Lebanese University, Beirut, Lebanon; ${ }^{5}$ Faculty of Public Health, La Sagesse University, Beirut, Lebanon; ${ }^{6} \mathrm{CERIPH}$, Center for Research in Public Health, Pharmacoepidemiology Surveillance Unit, Faculty of Public Health, Lebanese University, Fanar, Lebanon

Correspondence: Pascale Salameh Lebanese University, Pierre Gemayel Campus, Main street, Fanar, Lebanon Tel +96I 3385542

Email psalameh@ul.edu.lb
Background: Noncommunicable diseases are the leading cause of death in Lebanon, with cardiovascular diseases accounting for almost half of the annual deaths.

Purpose: We aimed to determine awareness of noncardiac Lebanese hospitalized patients for their coronary artery disease risk factors, their level of adherence to medications or lifestyle modifications, and assess factors associated with awareness.

Materials and methods: A cross-sectional study was conducted in 14 hospitals with a total of 382 patients. Levels of awareness were evaluated by the comparison of self-report with measurements and laboratory test results. Healthy behaviors and adherence to treatment were evaluated. Factors associated with better awareness were studied using multivariate regressions, while adherence to treatments and healthy lifestyle were described for the different risk factors and in the Framingham Risk Score categories.

Results: Our work revealed a moderate-to-high level of awareness ( $58.7 \%$ for overweight/obesity, $75 \%$ for hypertension, $85.7 \%$ for diabetes, and $86.4 \%$ for dyslipidemia) among patients for most cardiovascular risk factors, but a low-to-moderate level of adherence for some interventions such as physical exercise, weight loss, and smoking cessation.

Conclusion: The results emphasize on the importance of educational campaigns on healthy habits and screening to improve early diagnosis, increase patients' awareness of their risk factors, and, therefore, optimize primary prevention.

Keywords: coronary artery disease, risk factors, awareness, adherence, cardiovascular risk score

\section{Introduction}

Cardiovascular diseases (CVDs) are the leading cause of mortality worldwide, with around 17.7 million deaths in 2015, representing $31 \%$ of global mortality. Among CVD deaths, 7.4 million are due to coronary heart disease (CHD). More than three-quarters of CVD deaths occur in low- and middle-income countries, ${ }^{1}$ such as Lebanon. ${ }^{2}$ Overall, coronary artery disease (CAD) is increasing in the Middle East and Eastern Mediterranean countries due to the westernization of dietary habits, urbanization, technologic progress, and the reduction of physical activity. Multiple factors contribute to the pathogenesis of CVD. Although some factors are not modifiable (age, sex, and genetic predisposition to atherosclerotic disease), others, such as smoking, poor weight control, high blood pressure (HBP), diabetes, and dyslipidemia, can be modified or controlled. ${ }^{3}$ The likelihood of someone developing a cardiovascular event depends on a combination of risk factors (RFs) rather than the presence of one $\mathrm{RF}^{4}$ : the Framingham Risk Score (FRS) is a gender-specific algorithm used to estimate the 10-year cardiovascular risk (CVR) of an 
individual and classifies patients into low- $(<10 \%)$, intermediate- $(10 \%-20 \%)$, and high (>20\%)-risk groups. ${ }^{5,6}$

Awareness has been described as a prior knowledge of the status of cardiovascular risk factors (CVRFs). According to the Health Belief Model, an individual must perceive his or her health to be at risk or be susceptible to risk to take preventive action. ${ }^{7}$ The general awareness of chronic diseases including CVDs and their RFs can be a precondition for success in prevention and control of these diseases. This knowledge will inform individuals on healthy attitudes to adopt (diet, weight management, physical activity, and so on) and to be proactive in reducing their own lifetime risk by decreasing their exposure to modifiable CVRFs. ${ }^{4}$ The World Health Organization (WHO) has emphasized the role of health promotion and disease prevention as the most costeffective approaches to contain the CVD epidemic. ${ }^{8}$ Efforts to raise awareness of CVRF stress the importance of health promotion and disease prevention as important arenas to improve the quality of care and to contain the cost of health care in the Arab world. Many studies have shown low levels of patients awareness for their CVRFs. ${ }^{9,10}$ One of the main challenges in controlling heart disease is the lack of self-care, such as nonadherence to medications, unhealthy diet, and sedentary lifestyle, which leads to frequent hospitalizations. ${ }^{10}$

To our knowledge, few studies were conducted in Lebanon to assess public awareness of a particular $\mathrm{CVRF}^{11,12}$ or to make comparisons between urban and rural populations. ${ }^{13}$ In this context, our study was designed to measure the awareness of noncardiac hospitalized Lebanese patients about their coronary RFs (hypertension [HTN], dyslipidemia, diabetes, and excess weight), the adherence level to their treatments, and correlates of better adherence, and to identify factors associated with lack of awareness; we also calculated an FRS for each participant to assess compliance to healthy lifestyle behaviors among high-risk individuals.

\section{Materials and methods Study design}

A cross-sectional study was conducted in the two most populated Lebanese regions: Beirut and Mount-Lebanon. A list of all public and private hospitals in both governorates was obtained from the Ministry of Public Health. ${ }^{14}$ All hospitals were contacted, and 14 of 34 hospitals accepted to participate in the study, giving a participation rate of $41 \%$.

\section{Compliance with ethical standards}

Data collection was done in the following 14 hospitals: Sahel General Hospital, Al Zahraa University Hospital,
Saint George Hospital University Medical Center, Hopital Dr S. Serhal, St Joseph Hospital - Raymond \& Aida Najjar Medical Center, Hopital Hayek, Governmental Hospital Of Beirut Quarantine, Beirut Governmental University Hospital, Central Military Hospital, Hotel Dieu de France, Makassed, Mount Lebanon Hospital, Hopital Libanais, and Hopital Notre Dame Maritime. Before the interview, patients were informed about the objectives of the study and asked to give an oral consent. An ethical approval was obtained from all participating hospitals; they also approved of the verbal informed consent process used in our study.

\section{Study population}

The subjects enrolled were noncardiac Lebanese patients aged between 30 and 74 years who were hospitalized in the following services: general medicine, surgery, orthopedics, and maternity (recently given birth). Participants were considered as "cardiac" if a history of heart disease was indicated in their patient file (including myocardial infarction, stroke, unstable/stable angina, heart failure, cardiac arrhythmias). In addition to cardiac patients, those suffering from cancer, mental health problems, under chronic steroid treatment, unable to participate in an interview, as well as pregnant women were excluded.

\section{Sample size}

Sample size was calculated using Epi-info 7, assuming a CI of $95 \%$ and a margin of error of $5 \%$. In the absence of baseline data, we used an expected HTN awareness of 53\% (according to results from a previous study published in 2014). ${ }^{12}$ The minimal sample size necessary consisted of 382 subjects.

\section{Data collection}

Once approval was received from the ethics committee of each participating hospital, visits were scheduled with the hospital's administration. On visit days, a list of patients was administered to the investigator who chose a random sample to interview. Eligible patients who gave their consent to participate in the study were enrolled (Figure 1). Data collection took place from January to July 2017, using faceto-face interviews.

\section{Survey instrument}

The questionnaire used was inspired from the Healthy Heart Questionnaire $^{15}$ and included different sections. Section 1 contained questions about the different metabolic RFs (ie, HTN, diabetes, overweight/obesity, and dyslipidemia). In this section, participants were asked whether they have a 


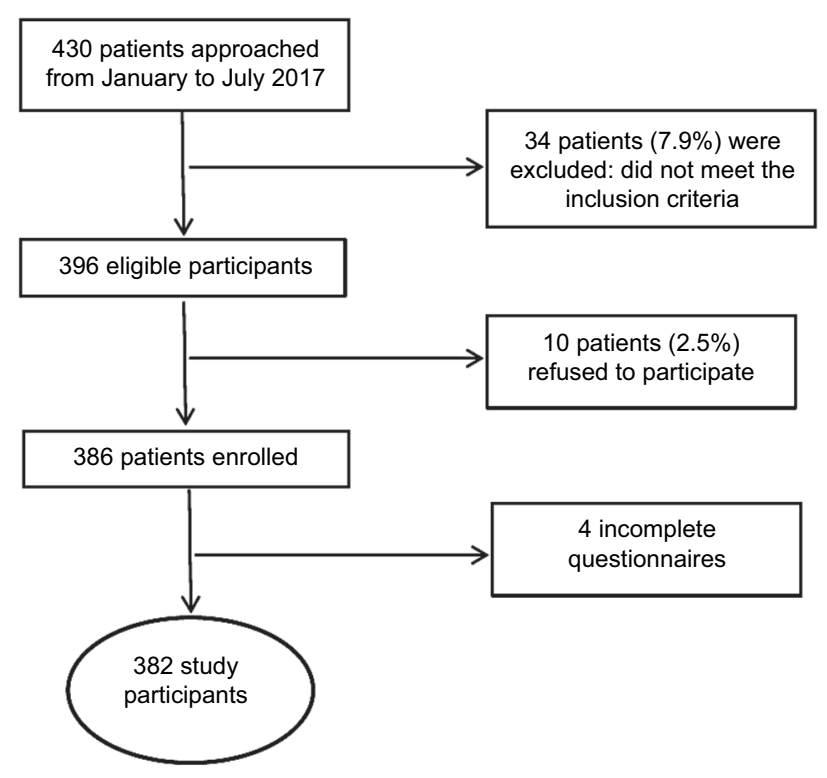

Figure I Patient flowchart.

personal or a family history of the RFs, whether they are on any medication related to the factor (if so, we asked about their adherence), and whether they are adopting appropriate preventive behaviors. In section 2, we asked details about behavioral RFs such as smoking and physical activity. Section 3 assessed sociodemographic characteristics, and the last section was reserved for the results of the last laboratory tests regarding fasting blood glucose and lipid panels. Finally, two blood pressure measurements were taken using a blood pressure monitor as recommended by JNC7 (seventh report of the joint national committee). ${ }^{16}$ The average of these two measurements was then used for the analysis. The survey instrument was first tested on $5 \%$ of the study population (20 subjects) and the necessary modifications were made subsequently.

\section{Outcomes assessment}

The presence of HTN, diabetes, and dyslipidemia was defined based on patient's self-report, the medications used, and laboratory results obtained from the patient's hospital records; the overweight/obesity status was defined as a body mass index (BMI) exceeding $25 \mathrm{~kg} / \mathrm{m}^{2}, 8$ "Lack of physical activity" was based on the following: 1) patients answering "No" to question "Do you exercise regularly?", and 2) patients exercising less than 30 minutes for at least 5 days/week. ${ }^{17}$

Patients were considered hypertensive if they answered "yes" to the question "Have you ever been told by a doctor or healthcare professional that you have HBP?" or if they were taking antihypertensive medications, or those with a BP $>140 / 90 \mathrm{mmHg}$, according to the current guidelines of Cardiology/European Atherosclerosis Society. ${ }^{16}$ Hypertensive patients were considered as aware of their HTN if they answered "yes" to first question.

Patients were considered as dyslipidemic if they answered "yes" to the question "have you ever been told by a doctor or healthcare professional that you have high blood lipids?" Or "yes" to question "are you currently taking lipid-lowering medications?" Or those with an abnormally high blood lipid concentration based on the lipid profile tested during hospitalization: hypercholesterolemia (total cholesterol $>200 \mathrm{mg}$ / $\mathrm{dL}$ ), hypoHDLemia (high-density lipoprotein cholesterol $<40$ $\mathrm{mg} / \mathrm{dL}$ ), hypertriglyceridemia (triglycerides $>150 \mathrm{mg} / \mathrm{dL}$ ), hyperLDLemia (low-density lipoprotein cholesterol $>130$ $\mathrm{mg} / \mathrm{dL}$ ) according to the current National Cholesterol Education Program guidelines. ${ }^{18}$ Hyperlipidemic patients were considered as aware of their dyslipidemia if they answered "yes" to first question.

Blood sugar was measured either by the hospital's professional team (fasting blood sugar [FBS]) or directly by the interviewer (random blood glucose $[\mathrm{RBS}]$ ). Patients were considered as diabetic if they answered "yes" to the question "have you ever been told by a doctor or healthcare professional that you have high blood sugar?" or those who were taking antidiabetic medications, or those with FBS $>126 \mathrm{mg}$ / dL (after 8 hours of fasting), or RBS $>200 \mathrm{mg} / \mathrm{dL}$, according to International Diabetes Federation guidelines and WHO recommendations. ${ }^{19}$ Diabetic patients were considered as aware of their diabetes if they answered "yes" to first question.

Participants suffering from any of these RFs were considered to be aware of their RFs if they had been informed by a doctor or health professional that they had the RFs.

Adherence to prevention interventions for each RF was described among patients who were aware of their RFs. Hypertensive patients who were aware of their HTN were asked if they were taking antihypertensive medications; if so, we asked them about their adherence using the following question: "Do you take your antihypertensive pills regularly and on time?"; patients were considered adherent if they replied to the question by "yes". They were also asked if they were trying to reduce their salt intake, if they exercised regularly (and the amount and intensity of activities), if they quit smoking (among hypertensive smokers), and if they were trying to lose weight (among overweight/obese hypertensive patients).

Diabetic patients who were aware of their diabetes were asked if they were taking antidiabetic medications (and 
whether it was done regularly and on time), if they were trying to reduce their sugar intake, if they exercised regularly (and the amount and intensity of activities), if they quit smoking (among diabetic smokers), and if they were trying to lose weight (among overweight/obese diabetic patients).

Patients suffering from dyslipidemia and who were aware of their condition were asked if they were on lipid-lowering drugs (and if they were adherent to the treatment), if they were trying to reduce their fat intake, if they exercised regularly (and the amount and intensity of activities), if they quit smoking (among smokers), and if they were trying to lose weight (among overweight/obese patients). Finally, overweight/obese participants were asked if they were taking preventive measures such as reducing their salt, sugar, and fat intakes, if they were exercising regularly (with the amount and intensity of activities), if they were still smoking (among smokers), and if they were trying to lose weight in order to prevent complications.

A comparison in the adherence to healthy behaviors was done between the different CVR groups obtained using the FRS Classification. ${ }^{6}$

\section{Statistical analysis}

Data were entered and analyzed using SPSS, version 21. A $P$-value $<0.05$ was considered significant. Categorical variables were described using frequencies and percentages, and continuous variables using means $( \pm \mathrm{SD})$. An appropriate bivariate analysis was performed for each explanatory variable with the awareness of HTN, diabetes, dyslipidemia, and excess weight considered as dependent variables. For continuous variables, independent-samples $t$-test was used for the comparison of two samples, and ANOVA test for more than two samples. For categorical variables, chi-square and Fisher's exact tests were used.

Multivariate logistic regressions were carried out for two dependent variables: awareness of HTN and awareness of excess weight, using a forward method and including in the models the independent variables that were associated with the dependent variables with a $P$-value $<0.20$ in the bivariate analysis. Multivariate analyses for diabetes and dyslipidemia were not done due to insufficient sample size regarding these two RFs.

\section{Results}

\section{Demographic characteristics}

This survey included 382 patients with an average age of $56.41 \pm 13.32$ years and a slight female predominance $(52.1 \%)$. In terms of age groups, the highest percentage was in patients aged $60-75$ (47.1\%vs $21.2 \%$ and $31.7 \%$ for age groups $30-44$ and 45-59, respectively) recruited mostly from Mount-Lebanon (66.5\%). Only $22.8 \%$ achieved a university level (Table 1 ).

\section{Prevalence and awareness of CVRFs}

Among studied parameters, the most common RF was insufficient regular physical activity $(85.1 \%)$ and the least prevalent was diabetes $(31.2 \%)$. The prevalence of other RFs is presented in Figure 2. The level of awareness was the highest in dyslipidemia (86.4\%), followed by diabetes (85.7\%); 75\% of hypertensive patients were aware of their HTN and only $58.7 \%$ were aware of their obesity or overweight (Figure 3 ).

\section{Factors associated with awareness of major RFs}

Table 2 shows the association of different factors with the awareness of the presence of HTN, diabetes, dyslipidemia,

Table I Baseline characteristics of study participants $(n=382)$

\begin{tabular}{|l|l|}
\hline Characteristics & N (\%) \\
\hline Age (mean \pm SD) & $56.41 \pm 13.32$ \\
Age groups, years & \\
30-44 years & $81(21.2)$ \\
$45-59$ years & $121(31.7)$ \\
$60-75$ years & $180(47.1)$ \\
Sex & \\
Male & $183(47.9)$ \\
Female & $199(52.1)$ \\
Province of residence & \\
Mount Lebanon & $254(66.5)$ \\
Beirut & $77(20.2)$ \\
North/Akkar & $22(5.8)$ \\
South Lebanon & $20(5.2)$ \\
Bekaa & $9(2.4)$ \\
Marital status & \\
Married & $289(75.7)$ \\
Divorced/widowed /not married & $93(24.3)$ \\
Employment status & \\
Full time & $145(38.0)$ \\
Part time & $6(1.6)$ \\
Unemployed & $147(38.5)$ \\
Retired & $84(22)$ \\
Educational level & \\
Illiterate & $54(14.1)$ \\
Primary education & $142(37.2)$ \\
Secondary education & $99(25.9)$ \\
University & $87(22.8)$ \\
Monthly income & \\
$<500$ USD & $69(18.1)$ \\
$500-1000$ USD & $174(45.5)$ \\
I000-2000 USD & $90(23.6)$ \\
2000-3000 USD & $36(9.4)$ \\
$>3000$ USD & $13(3.4)$ \\
\hline Note Cate
\end{tabular}

Note: Categorical variables are described as frequencies (percentages). 


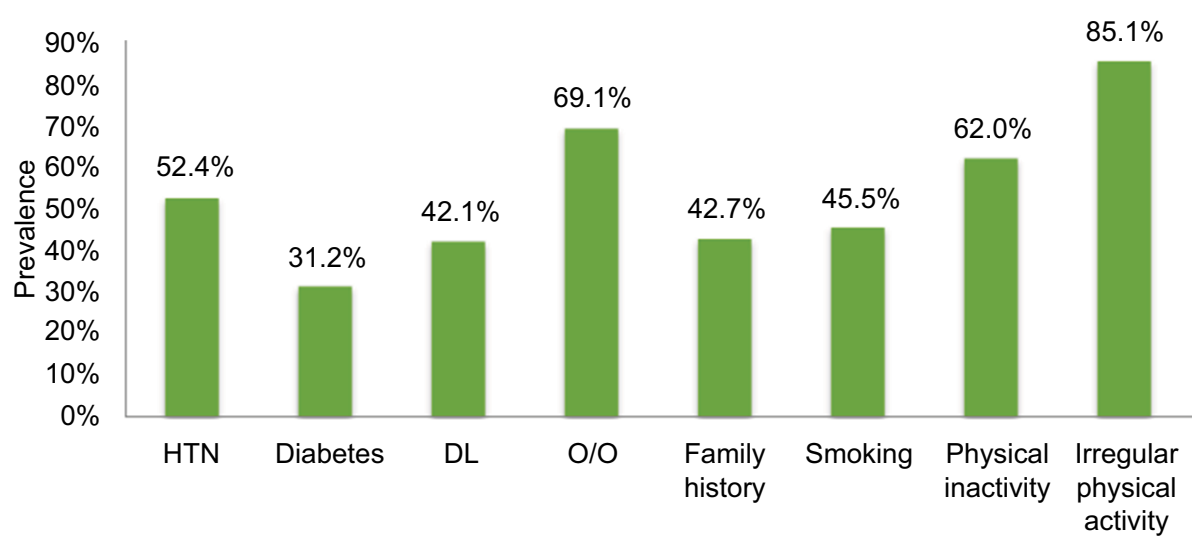

Figure 2 Prevalence of biologic, behavioral, and nonmodifiable risk factors in the study population. Abbreviations: DL, dyslipidemia; HTN, hypertension; O/O, overweight/obesity.

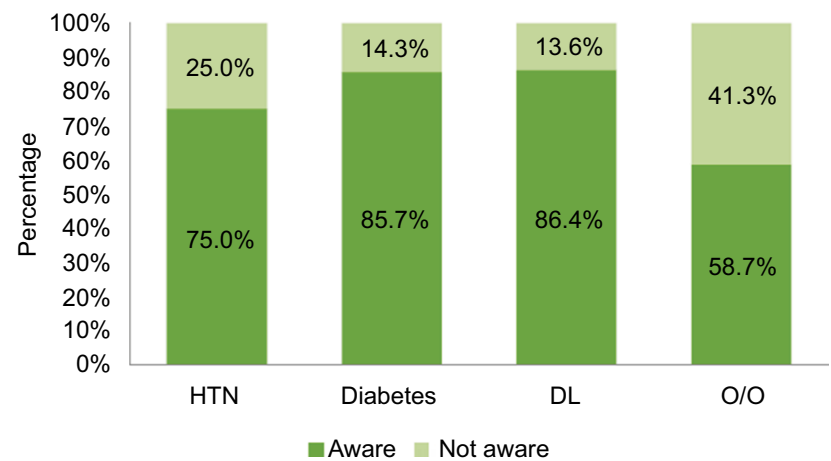

Figure 3 The awareness of various risk factors.

Abbreviations: DL, dyslipidemia; HTN, hypertension; O/O, overweight/obesity.

and excess weight. The awareness of hypertensive patients (150 subjects) varies significantly with age and sex, where subjects over 60 years and women were the most conscious. In addition, patients with dyslipidemia ( $85.9 \%$ vs $64.4 \%$ ), high BMI ( $83.9 \%$ for obese vs $67.9 \%$ for normal weight or underweight), or a family history of $\operatorname{HTN}(81.8 \%$ vs $60.3 \%)$ were significantly more conscious $(P<0.05)$. Awareness of the diabetic status (102 subjects) was significantly associated with the presence of dyslipidemia $(91.3 \%$ vs $78 \%, P=0.041)$ and a family history of diabetes $(92.7 \%$ vs $70.3 \%, P=0.001)$, whereas awareness of dyslipidemia (86.4\%) was significantly higher with age ( $42.9 \%$ vs $89.6 \%$ for age groups $30-44$ and 60-75 years, respectively, $P=0.013$ ) and with the presence of HTN (91.1\% vs $76.9 \%, P=0.034)$. Awareness of overweight/ obese patients (155 subjects) was significantly decreased with age ( $76.8 \%$ vs $48.8 \%$ for age groups $30-44$ and $60-75$ years, respectively), but increased in women (64.8\% vs $52.9 \%$ in men), and the level of education ( $67.2 \%$ vs $55.9 \%$ for secondary/higher education and illiterate, respectively).
Having a family history of HBP (64.1\% vs $50.9 \%)$ and being employee was significantly associated with awareness of overweight/obesity ( $65.7 \%$ vs $63.9 \%$ or $40 \%$ for employees and nonemployees or retired, respectively).

Two multivariable regressions on HTN and obesity awareness are presented in Table 3. Age appeared to be positively associated with the awareness of hypertensive status (adjusted odds ratio [aOR]: $1.083,95 \%$ CI: 1.057-1.110), with each year increasing the odds of being aware by $8 \%$; overweight people were also more aware of their HTN (aOR: 3.661, 95\% CI: $1.977-6.781)$. The place of residence was also significantly associated, with patients living outside Beirut and MountLebanon being associated with lower odds of awareness (aOR: 0.409, 95\% CI: 0.172-0.974). Respondents suffering from hyperlipidemia or having a family history of HTN were 2.089 times (95\% CI: $1.278-3.415)$ and 3.180 times (95\% CI: 1.848-5.471), respectively, more likely to report this CVRF. In the second regression, younger participants (aOR: 0.963, 95\% CI: 0.945-0.981) and female patients (aOR: 2.002, 95\% CI: 1.259-3.183) were the most aware of their excess weight.

\section{Adherence to treatments and risk prevention interventions}

The majority of patients with HTN, diabetes, and dyslipidemia and aware of their RFs were taking medications $(92.7 \%, 99 \%$, and $99 \%$, respectively), and were taking their medication on time $(85.6 \%, 89.9 \%$, and $83 \%$, respectively), while the rest were not adherent due to forgetfulness, the side effects, by personal choice, or due to the cost of drugs. As for preventive measures taken to reduce their risk of CVD, 78\% of hypertensive patients and $71 \%$ of the overweight were reducing their salt intake. Similarly, a reduction in sugar intake was noted among $89.2 \%$ of diabetics and $69 \%$ of overweight patients; 


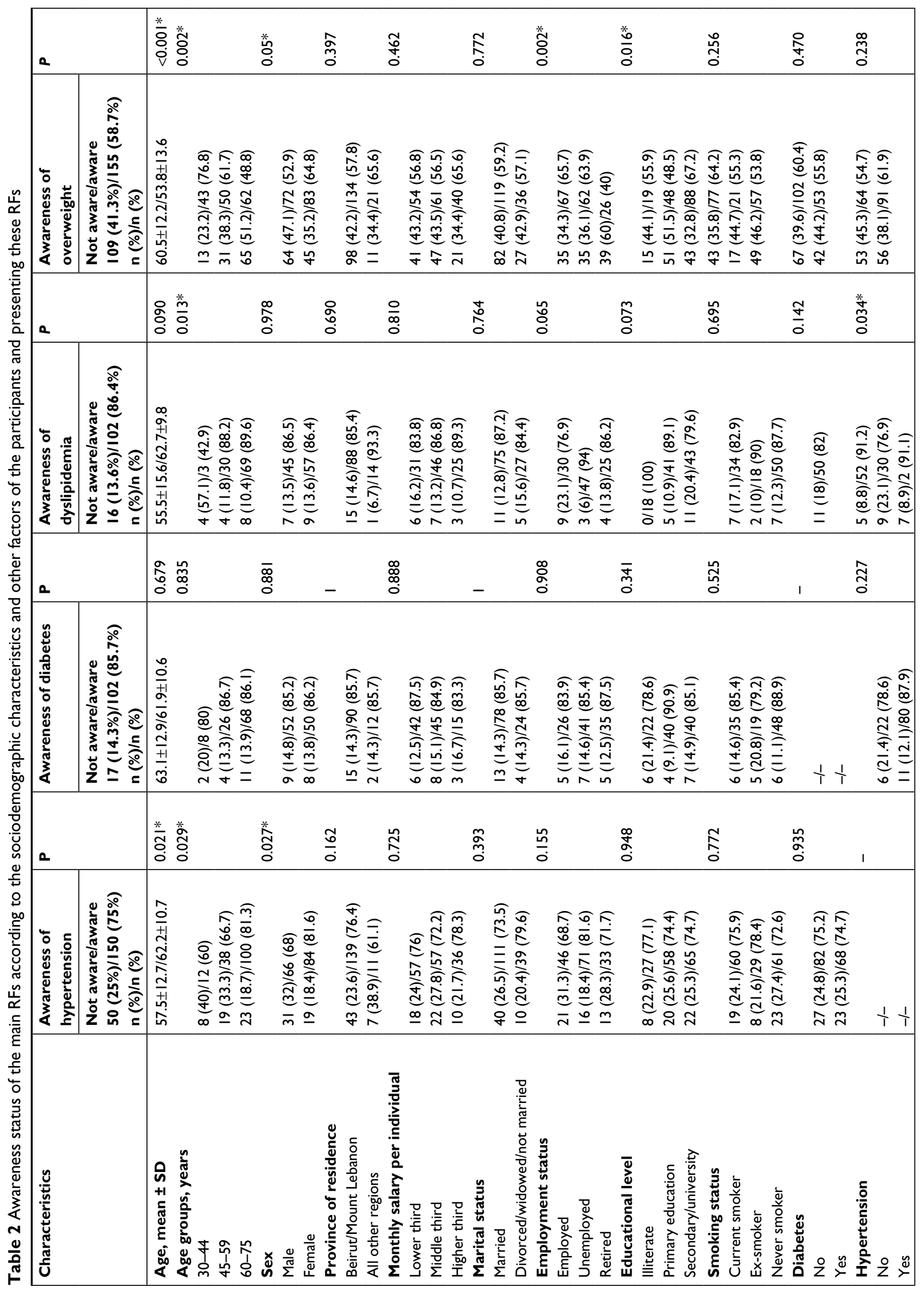




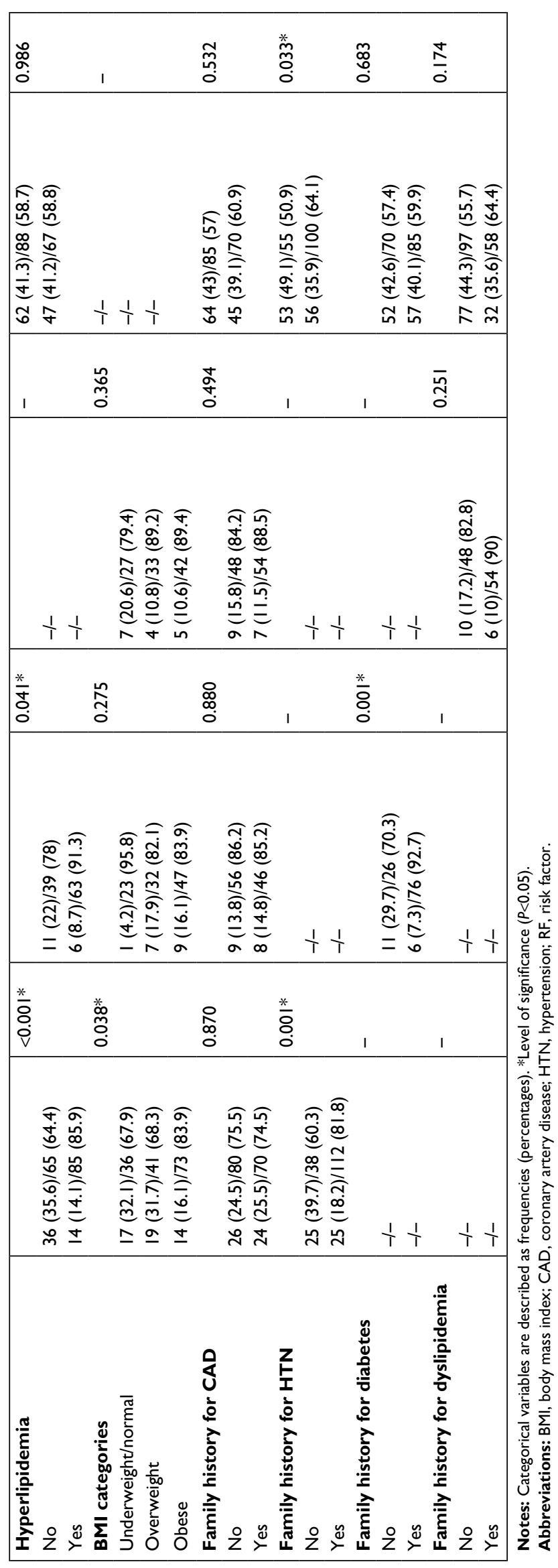

also, fat intake was reduced among $84.3 \%$ of dyslipidemic and $74.8 \%$ of overweight patients. However, only half of the patients with RFs stopped smoking. Overweight or obese subjects were trying to lose weight in $61.3 \%$ of cases. Most patients had poor adherence to either general or regular physical activity for 30 minutes at least 5 days/week, with, respectively, $32 \%$ and $12 \%$ among hypertensive, $26.5 \%$ and $10.8 \%$ among diabetics, $35.3 \%$ and $14.7 \%$ among dyslipidemic, and $33.5 \%$ and $11 \%$ among overweight/obese patients (Figure 4 ).

\section{CVRs and healthy behaviors}

Around half of the patients in the study (158 subjects, $41.4 \%$ ) had a high CVR compared to other groups (moderate: 95 subjects, $24.9 \%$, and low risk: 129 subjects, $33.8 \%$ ) based on the classification of FRS. ${ }^{6}$ Among the high CVR patients, the healthy behaviors that were most pursued were diets with low intakes of salt, sugars, and fats $(71.5 \%, 69 \%$, and $72.2 \%$, respectively). Only $17.7 \%$ of these patients had regular physical activity and $36.5 \%$ were trying to lose weight among overweight and obese patients. The comparison between the adhesion to healthy behaviors and different risk groups did not show any significant results (Table 4).

\section{Discussion}

Our study was designed to assess Lebanese patients' awareness of their CVRFs and their level of adherence to treatment and to healthy behaviors in public and private hospitals of Beirut and Mount-Lebanon. The majority of causes of heart attacks and strokes are usually the presence of a combination of RFs. ${ }^{1}$ Our analysis examined the awareness of six CVRFs: HBP, high cholesterol, diabetes, physical inactivity, and overweight/obesity. We found that awareness ranged from $58.7 \%$ for overweight status to $86.4 \%$ for dyslipidemia, with an adherence level to prevention insufficient for regular physical activity (10.8\%-14.7\%) and trying to lose weight (33.3\%-61.3\%); satisfactory for other interventions such as taking medications on time ( $83 \%-89.9 \%)$ and healthy diet $(69 \%-89.2 \%)$ among patients who have at least one RF and aware of this.

Lack of consciousness was analyzed in many previous studies, where it was shown that many patients were unaware of the CVRF they had. ${ }^{9,20}$ Interestingly, our results showed high level of awareness for most RFs, especially for diabetes $(85.7 \%)$ and dyslipidemia (86.4\%). This was higher than the percentages reported in China by Wang et al $(64.1 \%)^{21}$ and He et al $(11.6 \%)^{22}$ studies assessing awareness of diabetes and dyslipidemia, respectively, and higher than the rates of NHANES and CHARLS studies in the USA and China 
Table 3 Multivariable predictors on the awareness of hypertension and obesity

\begin{tabular}{|c|c|c|c|c|}
\hline Variables & $\begin{array}{l}\text { Awareness of } \\
\text { HTN aOR (95\% } \\
\text { CI) }\end{array}$ & $P$-value & $\begin{array}{l}\text { Awareness of } \\
\text { O/O aOR ( } 95 \% \\
\text { Cl) }\end{array}$ & $P$-value \\
\hline Age, years & $1.083(1.057-1.110)$ & $<0.00 I^{*}$ & $0.963(0.945-0.981)$ & $<0.001 *$ \\
\hline \multicolumn{5}{|l|}{ Sex } \\
\hline Female (vs male) & & & $2.002(1.259-3.183)$ & $0.003^{*}$ \\
\hline \multicolumn{5}{|l|}{ Province of residence } \\
\hline Other regions (vs Beirut/Mount & $0.409(0.172-0.974)$ & $0.043 *$ & & \\
\hline \multicolumn{5}{|l|}{ Lebanon) } \\
\hline Employment status & & $0.026 *$ & & \\
\hline Employed (reference) & I & & & \\
\hline Unemployed & $1.363(0.764-2.432)$ & 0.295 & & \\
\hline Retired & $0.543(0.266-1.106)$ & 0.092 & & \\
\hline \multicolumn{5}{|l|}{ Hyperlipidemia } \\
\hline Presence (vs absence) & $2.089(1.278-3.415)$ & $0.003 *$ & & \\
\hline BMI categories & & $<0.00 I^{*}$ & & \\
\hline Underweight/normal (reference) & I & & & \\
\hline Overweight & $1.253(0.662-2.369)$ & 0.488 & & \\
\hline Obese & $3.66 \mathrm{I}(\mathrm{I} .977-6.78 \mathrm{I})$ & $<0.00 I^{*}$ & & \\
\hline \multicolumn{5}{|l|}{ Family history for HTN } \\
\hline Presence (vs absence) & $3.180(|.848-5.47|)$ & $<0.00 I^{*}$ & & \\
\hline
\end{tabular}

Note: * Level of significance $(P<0.05)$.

Abbreviations: aOR, adjusted odds ratio; BMI, body mass index; HTN, hypertension; O/O, overweight/obesity.

where awareness reached $73.3 \%$ and $19.6 \%$ for dyslipidemia, respectively. ${ }^{23}$ One possible explanation for these discrepancies is that we restricted our study to hospitalized patients only. The high rate of awareness concerning dyslipidemia could also be due to the missing laboratory data for most patients (245 patients [64.1\%]).

Nearly $75 \%$ of subjects were aware of their HTN, which is higher than that reported by Matar et al study (53\%) of 2014 in Lebanon, ${ }^{12}$ which could be explained by several factors: first, the higher average age of our study population ( $56.41 \pm 13.32$ years vs $42.9 \pm 15.8$ years, respectively), which may be related to a higher probability of consciousness in these patients as higher age was noted as an associated factor to awareness. In fact, older participants could have more often come into contact with health systems. On the other hand, our sample consisted of hospitalized patients, which was not the case in their study. In addition, a difference between the places of residence of participants, where our study was concentrated in urban areas only, in which patients have better access to care compared to rural areas. ${ }^{13}$ When compared with adjacent countries, such as Turkey ${ }^{24}$ and Palestine, ${ }^{25}$ Lebanon had a higher awareness (75\%vs $40.7 \%$ and $51 \%)$. These differences may be explained by several factors, such as higher level of socioeconomic development, older age of Lebanese population, and higher ratio of physicians to the general population.
However, the awareness on obesity/overweight was $58.7 \%$, lower than the other RFs in our study, but still higher than the previous studies such as Andrikopoulos et al in Greece $(24.4 \%)^{26}$ and Sarriff et al in Malaysia (47.6\%), ${ }^{27}$ because the survey took place in urban areas without representatives from rural areas where awareness regarding health issues is likely to be lower. Similarly, women were significantly more aware of their overweight because women are generally more self-conscious about their physique than men, in line with other studies already conducted in Lebanon. ${ }^{28}$

Generally, adequate awareness of CVD and their RFs may help reduce the population's exposure to modifiable RFs and thereby contribute to prevention and control strategies. Awareness will help in molding the modifiable RFs in themselves and in those around them, as besides age, race, gender, and family history, all other RFs are modifiable. It was reassuring to see that our results obtained for adherence levels to treatment and prevention interventions were higher than those reported in another study conducted in Jordan, showing values of $20.9 \%$ and $72 \%$ for adherence to dietary regimes and drug treatments, respectively, but were lower for cessation smoking (70\%), and approximately similar for adoption of regular physical activity (16\%). ${ }^{29}$ Demographic, social, and cognitive factors, interactions between health care providers and patients, health system characteristics, medications involved, and overall patient 
A

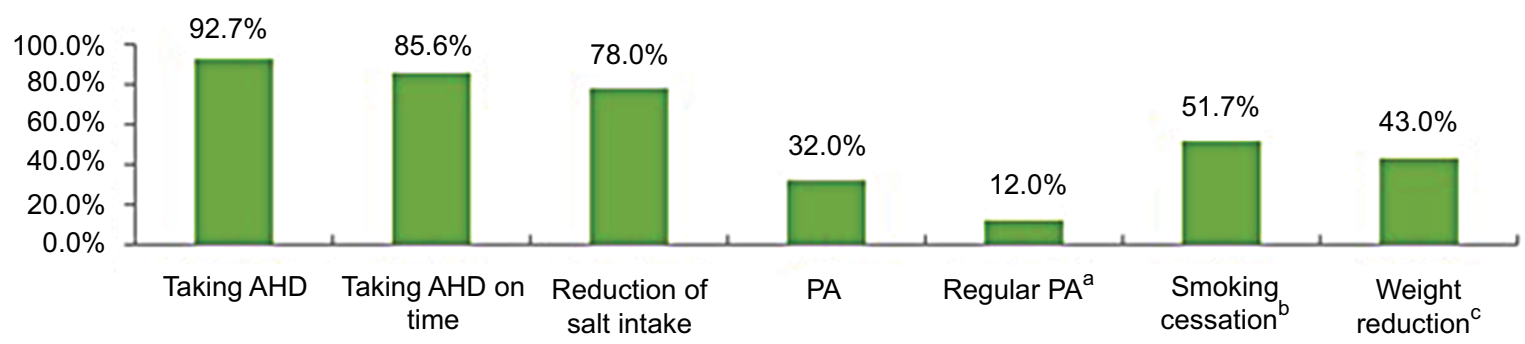

B

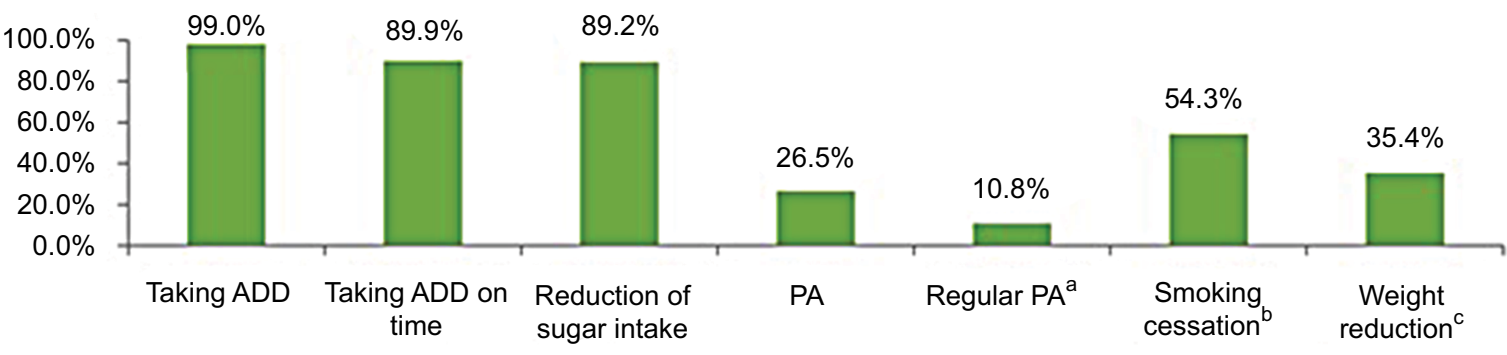

C

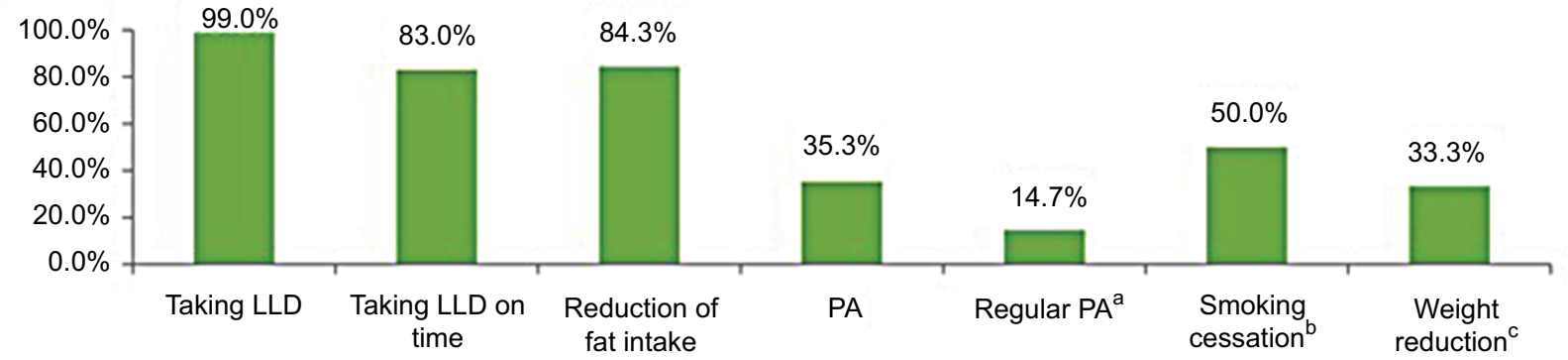

D

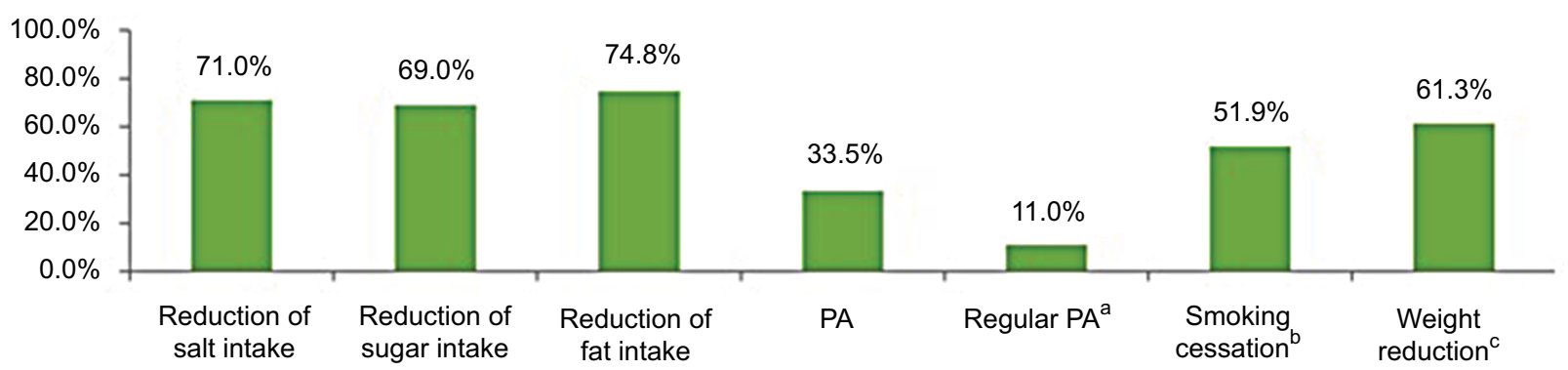

Figure 4 Adherence to treatment and prevention interventions.

Notes: (A) Among hypertensive patients and aware of their hypertension. (B) Among diabetic patients and aware of their diabetes. (C) Among dyslipidemic patients and

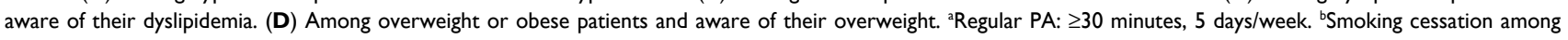
smokers. 'Weight reduction among overweight/obese patients.

Abbreviations: ADD, antidiabetic drugs; AHD, antihypertensive drugs; LLD, lipid-lowering drugs; PA, physical activity.

health may explain the variations in adherence rates among different populations.

Data from European epidemiologic studies from recent years indicate improvement in the control of some modifiable RFs like HTN and dyslipidemia or decrease in smoking prevalence leading to reduced CVD risk assessed by Framingham and European Systemic Coronary Risk Evaluation systems, which can be perceived as success of different community prevention programs, communication policies, and extensive work of numerous scientific associations. ${ }^{5}$ Thus, 
Table 4 Description of healthy behaviors among different risk groups $(n=382)$

\begin{tabular}{|l|l|l|l|}
\hline Healthy behaviors & $\begin{array}{l}\text { Lower risk, } \\
\mathbf{n}(\%) \\
\mathbf{I 2 9 ( 3 3 . 8 )}\end{array}$ & $\begin{array}{l}\text { Intermediate } \\
\text { risk, } \mathbf{n}(\%) \\
\mathbf{9 5}(\mathbf{2 4 . 9 )}\end{array}$ & $\begin{array}{l}\text { High risk, } \\
\mathbf{n}(\%) \\
\mathbf{1 5 8}(\mathbf{4 1 . 4 )}\end{array}$ \\
\hline Reduction of salt intake & $83(64.3)$ & $64(67.4)$ & $113(71.5)$ \\
Reduction of sugar intake & $84(65.1)$ & $58(61.1)$ & $109(69)$ \\
Reduction of fat intake & $97(75.2)$ & $77(81.1)$ & $114(72.2)$ \\
Physical activity & $51(39.5)$ & $30(31.6)$ & $64(40.5)$ \\
Regular physical activity $(\geq 30$ minutes, 5 days/week) & $21(16.3)$ & $29(42.6)$ & $28(17.7)$ \\
Weight reduction among O/O patients & $36(51.4)$ & $14(35)$ & $46(36.5)$ \\
Smoking cessation among smokers & $27(49.1)$ & $41(51.9)$ \\
\hline
\end{tabular}

Abbreviation: $\mathrm{O} / \mathrm{O}$, overweight/obese.

we performed an analysis by stratifying according to the FRS categories to see if patients classified as having a high CVR followed healthy lifestyle habits, according to WHO recommendations. ${ }^{8}$ But even in this group, healthy behaviors did not have high frequencies, and were not very different from those with low or moderate CVR. For this, there is a need for developing methods of changing the patients' attitude and practice in the prevention, treatment, and control of disease. Furthermore, the percentage of high CVR in our study was higher $(41.4 \%)$ than that demonstrated by Fahs et al, ${ }^{13}$ another study realized in Lebanon, where $13.9 \%$ of urban participants had an FRS of $>20 \%$, which could be explained by westernization of dietary habits, rapid or unplanned urbanization, technologic progress, and reduction of physical activity during work and leisure. ${ }^{3}$

\section{Public health recommendations}

The preventive health in Lebanon receives only sporadic attention in the context of medical visits for acute and chronic medical problems. On the basis of this study, emphasis should be placed on awareness of overweight and HTN for all age groups, both sexes, in persons with family histories, and especially those residents in rural areas. In addition, particular attention should be paid to lifestyle changes among Lebanese, targeting first those at high risk, to prevent and control the expected increase in the burden and mortality of CHD. Such an outcome underscores the importance of educational campaigns to healthy habits and screening as a strategy to improve early diagnosis, increase patients' awareness of RFs, and thus an effective primary prevention of CVD in general population. This could possibly reduce the burden of CAD in Lebanon.

Hence, appropriate actions should be done not only in Lebanon but in neighboring countries. ${ }^{24,25}$ Several reports addressed this issue, among these, the world health report $2002,{ }^{8}$ which targets a healthier future worldwide and tries to reverse the epidemic of CVD. Among the recommenda- tions that should be applied especially in Lebanon are the development of effective policies for the prevention of high risks to health, such as smoking, unhealthy diet, physical inactivity, and obesity; implementing new intervention programs targeting risk prevention, for all age groups, and especially for high-risk groups; and the improvement of data sources and systems to monitor key indicators relevant to CVD prevention.

\section{Strengths and limitations of the study}

This study has several strengths. It is the first study in Lebanon to assess awareness of CVRFs in a representative sample of noncardiac hospitalized patients, as well as their level of adherence to drug and non-drug treatments for biologic RFs and other prevention strategies. Also, the double measurement of BP reduced the "white coat" effect and the glucose measurement (RBS) provided values for all participants to measure their awareness. In addition, the level of awareness of different RFs was analyzed according to several social and demographic factors and the presence of other RFs. Nevertheless, like any epidemiologic investigation, our study has certain limitations that should be reported. First of all, the survey contains a selection bias because the sample was chosen among hospitalized patients, and the results of study could be generalizable on hospitalized patients of the regions of Beirut and Mount-Lebanon, but not on the general population. A more large-scale study with a larger sample size could be beneficial to the generalization of the results. The assessment of behavioral RF and preventive measures (such as physical activity, smoking cessation, and lowering intake of salt and sugar) were self-reported. Overreporting of healthy behaviors and underreporting of negative ones due to social desirability could have led to an information bias through possible misclassifications. Thus, one could expect that the real situation is even worse than the observed findings. A memory bias would also be present. Also, waist 
circumference, which has been correlated with the development of $\mathrm{CAD},{ }^{30}$ could not be measured for the majority of patients because of their inability to move. Finally, a classification bias of the dyslipidemia awareness would be present because lipid profile results were only obtained for $1 / 5$ of all patients, which could have introduced an overestimation of the percentage of dyslipidemia' awareness.

\section{Conclusion}

Our study showed a satisfactory level of awareness in Lebanese patients for most CVRF, but a low and moderately high level of adherence for some prevention interventions such as exercise, testing weight loss, and smoking cessation. Although awareness, adherence, and control rates are better than other countries, they remain low and should be substantially improved.

\section{Acknowledgments}

We would like to thank all our participants who shared their personal and intimate information with us. In addition, to all the hospitals' administrations that accepted to participate in the study. Grateful thanks and recognition to Dr Haitham Abed Al Rahman for his involvement and coordination.

\section{Authors contribution}

Fatima Ghaddar contributed toward study conception and data collection. Rouba K Zeidan contributed toward study conception and project supervision. All authors contributed toward data analysis, drafting and revising the paper, gave final approval of the version to be published, and agree to be accountable for all aspects of the work.

\section{Disclosure}

The authors report no conflicts of interest in this work.

\section{References}

1. WHO. Cardiovascular diseases (CVDs); 2015. Available from: http:// www.who.int/mediacentre/factsheets/fs317/en/. Accessed April 9, 2018

2. World Bank Country and Lending Groups - World Bank Data Help Desk. Available from: https://datahelpdesk.worldbank.org/knowledgebase/articles/906519-world-bank-country-and-lending-groups. Accessed January 14, 2017.

3. Alwan AAS, Agis T, Organisation mondiale de la santé. Global Status Report on Noncommunicable Diseases 2010. Geneva: World Health Organization; 2011.

4. Fifth Joint Task Force of the European Society of Cardiology, European Association of Echocardiography, European Association of Percutaneous Cardiovascular Interventions. European Guidelines on cardiovascular disease prevention in clinical practice (version 2012): the Fifth Joint Task Force of the European Society of Cardiology and Other Societies on Cardiovascular Disease Prevention in Clinical Practice (constituted by representatives of nine societies and by invited experts. Eur J Prev Cardiol. 2012;19(4):585-667.
5. Karam C, Beauchet A, Czernichow S, et al. Trends in cardiovascular disease risk factor prevalence and estimated 10 -year cardiovascular risk scores in a large untreated French Urban population: the CARVAR 92 Study. PLoS One. 2015;10(4):e0124817.

6. Framingham Heart Study. Available from: https://www.framinghamheartstudy.org/fhs-risk-functions/cardiovascular-disease-10-year-risk/. Accessed April 24, 2018.

7. Jones CL, Jensen JD, Scherr CL, Brown NR, Christy K, Weaver J. The Health belief model as an explanatory framework in communication research: exploring parallel, serial, and moderated mediation. Health Commun. 2015;30(6):566-576.

8. World Health Organization. The World Health Report 2002: Reducing Risks, Promoting Healthy Life. Geneva: World Health Organization; 2002.

9. Pan L, Yang Z, Wu Y, et al. The prevalence, awareness, treatment and control of dyslipidemia among adults in China. Atherosclerosis. 2016;248:2-9.

10. Heydari A, Ziaee ES, Gazrani A. Relationship between awareness of disease and adherence to therapeutic regimen among cardiac patients. Int J Community Based Nurs Midwifery. 2015;3(1):23-30.

11. Farah R, Zeidan RK, Chahine MN, et al. Predictors of uncontrolled blood pressure in treated hypertensive individuals: first population-based study in Lebanon. J Clin Hypertens. 2016;18(9):871-877.

12. Matar D, Frangieh AH, Abouassi S, et al. Prevalence, awareness, treatment, and control of hypertension in Lebanon. J Clin Hypertens. 2015;17(5):381-388.

13. Fahs I, Khalife Z, Malaeb D, Iskandarani M, Salameh P. The prevalence and awareness of cardiovascular diseases risk factors among the Lebanese population: a prospective study comparing urban to rural populations. Cardiol Res Pract. 2017;2017:1-10.

14. Lebanese Ministry of Public Health-moph-Health Facility Locator. Available from: http://www.moph.gov.lb. Accessed February 28, 2018.

15. General Population - Pre-Test - Healthy Heart Questionnaire (HHQGP-1). Available from: http://www.ucdenver.edu/academics/colleges/ PublicHealth/research/centers/CAIANH/ceed/Documents/General\%20 Population\%20-\%20Pre-Test $\% 20-\% 2010-12-09$ Clean.pdf. Accessed February 28, 2018.

16. Chobanian AV, Bakris GL, Black HR, et al. Seventh report of the Joint National Committee on Prevention, Detection, Evaluation, and Treatment of High Blood Pressure. Hypertension. 2003;42(6): $1206-1252$.

17. Garber CE, Blissmer B, Deschenes MR, et al. American College of Sports Medicine position stand. Quantity and quality of exercise for developing and maintaining cardiorespiratory, musculoskeletal, and neuromotor fitness in apparently healthy adults: guidance for prescribing exercise. Med Sci Sports Exerc. 2011;43(7):1334-1359.

18. National Cholesterol Education Program (NCEP) Expert Panel on Detection, Evaluation, and Treatment of High Blood Cholesterol in Adults (Adult Treatment Panel III). Third Report of the National Cholesterol Education Program (NCEP). Expert Panel on Detection, Evaluation, and Treatment of High Blood Cholesterol in Adults (Adult Treatment Panel III) final report. Circulation. 2002;106(25):3143-3421.

19. World Health Organization, International Diabetes Federation. Definition and Diagnosis of Diabetes Mellitus and Intermediate Hyperglycaemia: Report of a WHO/IDF Consultation.; 2006. Available from: http://www.who.int/diabetes/publications/diagnosis_diabetes2006/en/. Accessed February 28, 2018.

20. Deepa M, Bhansali A, Anjana RM, et al. Knowledge and awareness of diabetes in urban and rural India: The Indian Council of Medical Research India Diabetes Study (Phase I): Indian Council of Medical Research India Diabetes 4. Indian J Endocrinol Metab. 2014;18(3):379.

21. Wang C, Yu Y, Zhang X, et al. Awareness, treatment, control of diabetes mellitus and the risk factors: survey results from northeast China. PLoS One. 2014;9(7):e103594.

22. He H, Yu YQ, Li Y, et al. Dyslipidemia awareness, treatment, control and influence factors among adults in the Jilin province in China: a cross-sectional study. Lipids Health Dis. 2014;13(1):122. 
23. Lu Y, Wang P, Zhou T, et al. Comparison of prevalence, awareness, treatment, and control of cardiovascular risk factors in China and the United States. J Am Heart Assoc. 2018;7(3):e007462.

24. Altun B, Arici M, Nergizoğlu G, et al. Prevalence, awareness, treatment and control of hypertension in Turkey (the PatenT study) in 2003. J Hypertens. 2005;23(10):1817-1823.

25. Khdour MR, Hallak HO, Shaeen M, Jarab AS, Al-Shahed QN. Prevalence, awareness, treatment and control of hypertension in the Palestinian population. J Hum Hypertens. 2013;27(10):623-628.

26. Andrikopoulos G, Richter D, Sakellariou D, et al. High prevalence and diminished awareness of overweight and obesity in a Mediterranean population. An alarming call for action. Open Cardiovasc Med J. 2012;6(1): 141-146.
27. Sarriff A, Amin AM, Mostafa H. Public knowledge and awareness of cardiovascular diseases and the expected role of community pharmacists in the prevention and management of cardiovascular diseases in Penang, Malaysia. Chiang Mai Univ J Nat Sci. 2014;13(3):355-369.

28. Nasreddine L, Hwalla N, Sibai A, Hamzé M, Parent-Massin D. Food consumption patterns in an adult urban population in Beirut, Lebanon. Public Health Nutr. 2006;9(2):194-203.

29. Mosleh SM, Darawad M. Patients' adherence to healthy behavior in coronary heart disease: risk factor management among Jordanian patients. J Cardiovasc Nurs. 2015;30(6):471-478.

30. Canoy D, Cairns BJ, Balkwill A, et al. Coronary heart disease incidence in women by waist circumference within categories of body mass index. Eur J Prev Cardiol. 2013;20(5):759-762.

\section{Publish your work in this journal}

Vascular Health and Risk Management is an international, peerreviewed journal of therapeutics and risk management, focusing on concise rapid reporting of clinical studies on the processes involved in the maintenance of vascular health; the monitoring, prevention and treatment of vascular disease and its sequelae; and the involvement of metabolic disorders, particularly diabetes. This journal is indexed on PubMed Central and MedLine. The manuscript management system is completely online and includes a very quick and fair peer-review system, which is all easy to use. Visit http://www.dovepress.com/ testimonials.php to read real quotes from published authors. 